\title{
Comparison between Edge Detection Methods on UTeM Unmanned Arial Vehicles Images
}

\author{
Zuraini Othman*, Asmala Ahmad, Fauziah Kasmin, Sharifah Sakinah Syed Ahmad, Mohd Yazid Abu Sari \\ and Muhammad Amin Mustapha.
}

Department of Intelligent Computing and Analytics, Faculty of Information \& Communication Technology, Universiti Teknikal Malaysia Melaka, Hang Tuah Jaya, 76100 Durian Tunggal,Melaka, Malaysia.

\begin{abstract}
Machine vision calls for the use of detectors to ascertain the features and type of object portrayed in the image. The employment of unmanned aerial vehicles (UAVs), which can function freely in active and precarious settings, is currently gaining momentum. These vehicles are mainly used for the detecting, classifying and tracking of an object. However, the achievement of these objectives necessitates the involvement of an effective edge detection procedure. Sobel, Canny, Prewitt and LoG are among the many edge detection procedures presently available. In this endeavour, we opted for the utilization of UTeM UAVs images for an evaluation of these edge detection procedures. During our investigations, the ground truth edge images were corroborated by a specialist in this field. The results obtained from these investigations revealed that in terms of accuracy, precision, sensitivity and f-measure, the Prewitt procedure outperforms the other methods mentioned.
\end{abstract}

\section{Introduction}

When it comes to machine vision, the analysis of an image is significantly determined by the particulars related to its edges. Essentially, edge detection is a technique employed to identify the alterations that transpire at the pixel of an image. The mathematical procedures related to edge detection are aimed at distinguishing the points in a digital image at which the luminosity of the image varies abruptly (or put differently, has discontinuities). These points are characteristically structured in an array of arched line segments known as edges. The term step detection denotes the search for discontinuities in one-dimensional signals, while the term change detection denotes the search for signal discontinuities over time. Edge detection is an indispensable instrument for image processing, machine vision and computer vision. This is especially so in matters related to the detection and extraction of features [1].

The acquiring of precise images is especially important in the areas of biometrics, iridology, manufacturing, medical procedures and UAVs [2]-[6]. Precise images serve to ensure that the procedures employed in these areas are appropriate from start to finish.

Edge detection techniques, including those mentioned in [7]-[11], have been the subject of many investigations. Among the techniques available, the most frequently employed are Sobel, Prewitt, Canny, and Laplacian of Gaussian (LoG). Studies conducted on the Canny technique [12], [13] have served to improve its performance in the context of iris, scenery, retina and handwritten images.

This endeavour forwards an innovative UAVs image dataset for the site of Universiti Teknikal Malaysia Melaka (UTeM). This dataset is exceptional as it holds a wide range of features that include buildings, highways and oil farms and nature around UTeM. The edge image ground truth is provided for every image present in this dataset. We also discussed and evaluated the Sobel, LoG, Canny and Prewitt edge detection procedures.

\section{Edge Detection}

An edge, in the context of an image, is an arc that tracks a route of quick alterations in the intensity of the image. More often than not, edges concern the borders of objects in a setting. The task of edge detection is to make out the edges of an image. In the case of a digital image, the edge is a compilation of pixels with grey values that come with a step or roof change. Here, the edge also denotes the segment where the luminosity of the local area of the image varies considerably. Generally, this is attributed to the swift alteration of a grey value into a substantially different grey value within a small buffer area. Edges are frequently detectable between objects and backgrounds, objects and objects, as well as between primitives and primitives. The edge of an object is revealed by the lack of continuity in its grey profile. This circumstance provides a route towards edge detection. Edge detection involves a scrutiny of the alterations of a sole image pixel in a grey area, and the subsequent employment of the edge neighbouring first order or second order to discern the edge. This process is known as the local operator edge detection procedure. Basically, edge detection has to do with the gauging, distinguishing and locating of alterations in the grey profile of an image. The most fundamental and noticeable feature of any image is its edge and line. The composition of the edge and line in an image reveals the structure of an object. This elevates the importance of edge detection in the realms of graphics processing and feature extraction [14].

\footnotetext{
*Corresponding author: zuraini@utem.edu.my
} 
The employment of four edge detection techniques is described below.

\subsection{Canny Method}

The Canny method was put forward by [15]. ]. Listed below are the steps for the tradition Canny algorithm [16], [17].

- The Gaussian filter is utilized to smooth the image in order to restrain noise.

- Finite difference on the first order partial derivative is used to work out the gradient magnitude $M(x, y)$ as well as the gradient direction $H(x, y)$ of the image. $M(x, y)$ is computed through the equation $M(x, y)=\sqrt{E_{x}(x, y)^{2}+E_{y}(x, y)^{2}}$,

while $\mathrm{H}(\mathrm{x}, \mathrm{y})$ is computed through the equation

$$
H(x, y)=\arctan \left(E_{x}(x, y) / E_{y}(x, y)\right)
$$

in which $E_{x}$ and $E_{y}$ portray the image subsequent to the influence of the filter alongside the row-column route.

- Non-maximum suppression is applied for the gradient magnitude.

- The dual-threshold algorithm is utilized to distinguish and link the edges.

- In order to ascertain the final edges, hysteresis is employed to hold back any edge not linked to an exceptionally durable edge.

\subsection{The Sobel method}

As displayed in Figure 1, the operator comprises two $3 \times 3$ convolution kernels. Essentially, one kernel is a depiction of the other turned around by $90^{\circ}$.

\begin{tabular}{|l|l|l|}
\hline-1 & 0 & +1 \\
\hline-2 & 0 & +2 \\
\hline-1 & 0 & +1 \\
\hline
\end{tabular}

Gx

\begin{tabular}{|c|c|c|}
\hline+1 & +2 & +1 \\
\hline 0 & 0 & 0 \\
\hline-1 & -2 & -1 \\
\hline
\end{tabular}

Gy
Fig. 1. Sobel convolution kernels

These kernels are conceived for maximum reaction to the edges that stretch in a perpendicular and parallel line in relation to the pixel grid. Each of the two perpendicular orientations is represented by one kernel. The application of the kernels to the input image can be carried out independently to realize distinct gradient component dimensions for each orientation (represented as $G x$ and $G y$ ). $G x$ and $G y$ can then be merged to ascertain both the gradient's absolute magnitude at each point, as well as its orientation. The gradient magnitude is calculated by way of the equation

$$
|G|=\sqrt{G x^{2}+G y^{2}}
$$

More often than not, an estimated magnitude is derived through the equation

$$
|G|=|G x|+|G y|
$$

which facilitates a quicker computation. The angle of orientation of the edge (in relation to the pixel grid) which yields the spatial gradient is calculated through the equation

$$
\theta=\arctan (G y / G x)
$$

\subsection{The Prewitt method}

The Prewitt operator, which is comparable to that of the Sobel operator, is applied for the detection of vertical and horizontal edges in an image.

$$
h_{1}=\left[\begin{array}{ccc}
1 & 1 & 1 \\
0 & 0 & 0 \\
-1 & -1 & -1
\end{array}\right] \quad h_{3}=\left[\begin{array}{ccc}
-1 & 0 & 1 \\
-1 & 0 & 1 \\
-1 & 0 & 1
\end{array}\right]
$$

Fig. 2. Prewitt convolution kernels

\subsection{The LoG method}

The Laplacian can be described as a 2-D isotropic calculation of an image's 2nd spatial derivative. The Laplacian of an image draws attention to areas of quick intensity alterations. Frequently employed for edge detection, the Laplacian is regularly used for an image that has undergone smoothing by way of a device resembling a Gaussian smoothing filter. This smoothing process is for the purpose of decreasing the sensitivity of the image to noise. Generally, the operator harnesses one grey level image as input, and generates a separate grey level image as output.

The Laplacian $L(x, y)$ of an image with pixel intensity values $I(x, y)$ is depicted as:

$$
L(x, y)=\frac{\partial^{2} I}{\partial x^{2}}+\frac{\partial^{2} I}{\partial y^{2}}
$$

An estimation of the second derivatives in the definition of the Laplacian calls for the involvement of a separate convolution kernel. This is due to the fact that the input image is portrayed as a collection of distinct pixels. Displayed in Figure 3 below are three frequently employed small kernels.

\begin{tabular}{|c|c|c|}
\hline 0 & 1 & 0 \\
\hline 1 & -4 & 1 \\
\hline 0 & 1 & 0 \\
\hline
\end{tabular}

\begin{tabular}{|c|c|c|}
\hline 1 & 1 & 1 \\
\hline 1 & -8 & 1 \\
\hline 1 & 1 & 1 \\
\hline
\end{tabular}

\begin{tabular}{|c|c|c|}
\hline-1 & 2 & -1 \\
\hline 2 & -4 & 2 \\
\hline-1 & 2 & -1 \\
\hline
\end{tabular}

Fig. 3. LoG kernel

The fact that these kernels are estimating a second derivative measurement on the image renders them exceedingly susceptible to noise. To overcome this setback, the image is usually Gaussian smoothed prior to the introduction of the Laplacian filter. This preprocessing intervention serves to lessen the high 
frequency noise elements before the onset of the differentiation stage.

As the convolution operation is deemed associative, it is possible to initially coil the Gaussian smoothing filter and the Laplacian filter together. The resulting hybrid filter is then coiled with the image to acquire the targeted result. This move comes with two benefits:

- As both the Gaussian and Laplacian kernels are typically much slighter than the image, this process entails significantly less mathematical activities.

- The LoG kernel can be recomputed beforehand so that the performance of only a single runtime convolution is required on the image.

The focus of the 2-D LoG function is on zero with the Gaussian standard deviation $\sigma$ depicted as:

$$
\operatorname{LoG}(x, y)=-\frac{1}{\pi \sigma^{4}}\left[1-\frac{x^{2}+y^{2}}{2 \sigma^{2}}\right] e^{-\frac{x^{2}+y^{2}}{2 \sigma^{2}}}
$$

During this investigation, the Canny, Sobel, Prewitt and LoG edge detection methods was applied for each UTeM UAVs image. Subsequently, the results attained were weighed against the related ground truth images to determine the value of the measurement.

\section{Methodology}

A Walkera quad copter UAVs was employed to gather data for this study [18]. This UAVs has a dimension of $620 \times 620 \times 460 \mathrm{~mm}$ and weighs $3900 \mathrm{~g}$. Powered by a LiPo 6S $10000 \mathrm{mAh}$ battery, it comes with GPS mechanisms to facilitate controlling and tracking functions. It is also furnished with a RGB digital camera for the gathering of basic data in the form of aerial images. During its foray for data, the UAVs flew at a velocity of roughly $3 \mathrm{~ms}^{-1}$ and a height close to $100 \mathrm{~m}$. The location for this study is the main UTeM campus in Durian Tunggal, Melaka, Malaysia. To be more precise, it is sited at a latitude and longitude of $2.3139^{\circ} \mathrm{N}$ and $102.3212^{\circ}$ E respectively. Mission Planner software was employed to plot the navigation route for the gathering of images featuring buildings of interest.

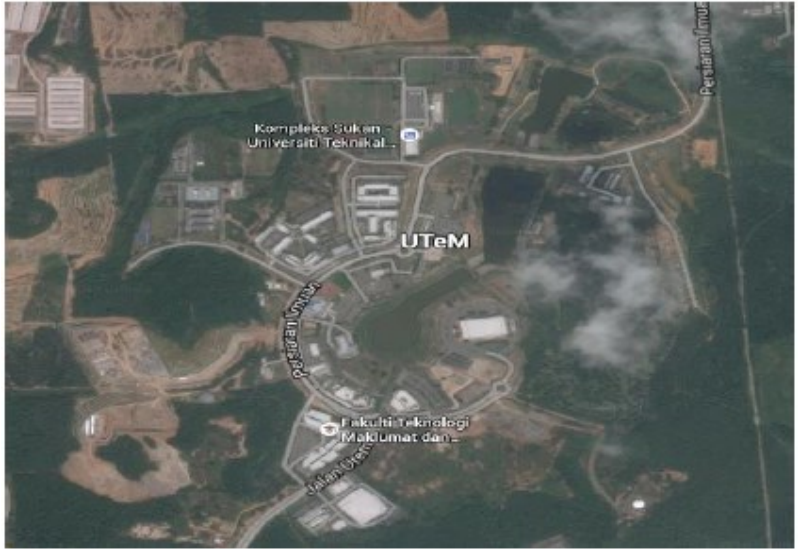

Fig. 4. Study location (Google Maps, 2017).

At the outset, for every building image captured, a reference edge image of the building was drawn by hand. This was achieved by way of a visual inspection of the edges existing in the initial image.

\subsection{Comparison between Edge Detection Methods on UTeM Unmanned Arial Vehicle Images}

Every image acquired was transformed into a grey scale image. For each image, edge ground images are generated by hand and subsequently validated by a specialist in the geospatial field. The workflow for our recommended procedure is exhibited in Figure 5.

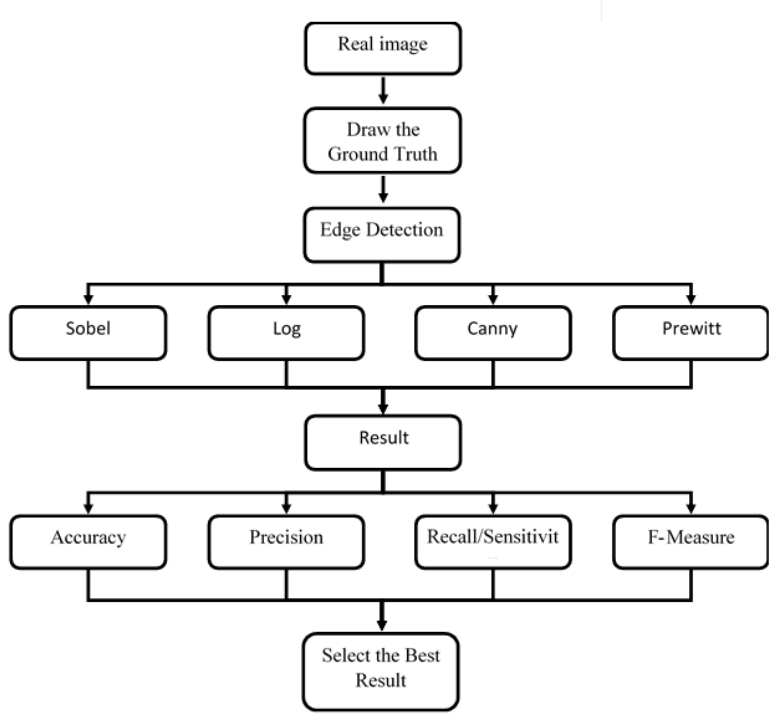

Fig. 5. Recommended procedure

In order to facilitate the computed result display, a graphical user interface (GUI) was developed for comparison. This is illustrated in Figure 6. 


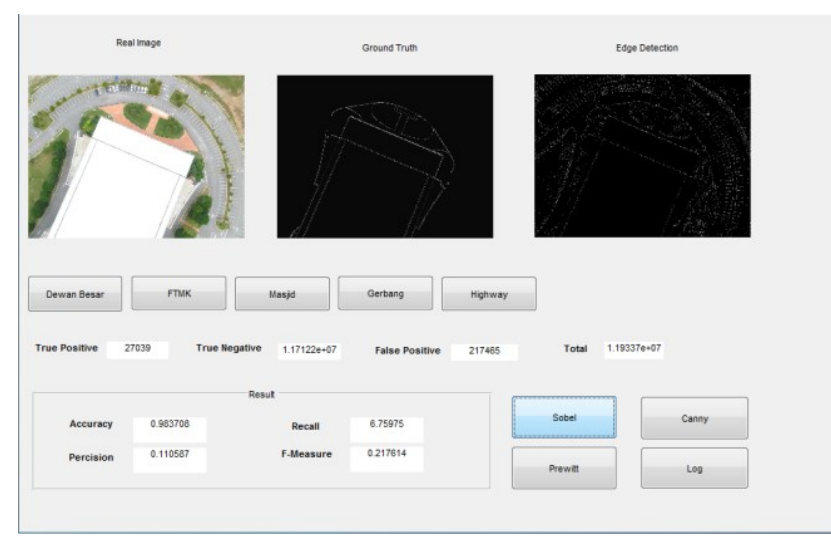

Fig. 6. Utilization of GUI

\section{Results and Discussion}

The measurement procedures for the results breakdown are as follows:

Accuracy $=\frac{\sum \text { True Positive }+\sum \text { True Negative }}{\sum \text { Total Population }}$

Precision $=\frac{\sum \text { True Positive }}{\sum \text { Prediction Positive }}$

Recall / Sensitivity $=\frac{\sum \text { True Positive }}{\sum \text { Condition Positive }}$

F-Measure $=2 \times\left(\frac{(\text { Precision } \times \text { Recall })}{(\text { Precision }+ \text { Recall })}\right)$

Figure 7 provides examples of the UTeM UAVs images dataset. As can be observed, original images of the main hall, mosque and highway are displayed with their corresponding edge images as a ground truth.

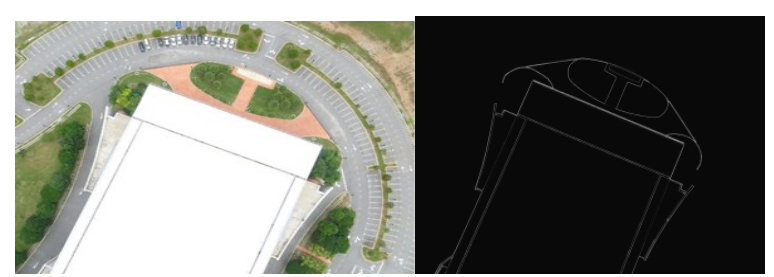

(a)
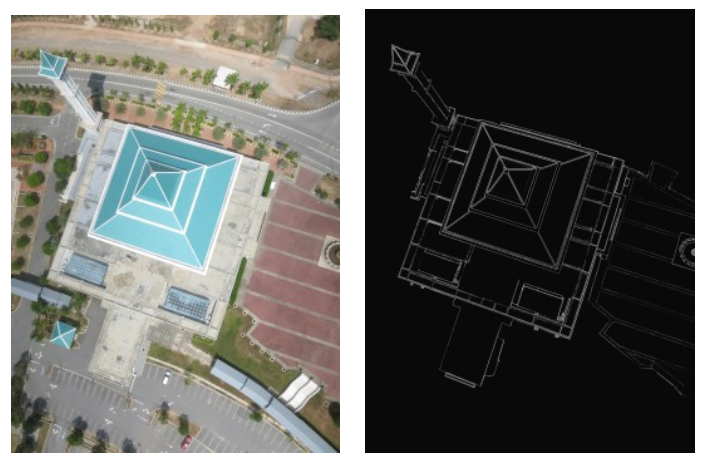

(b)
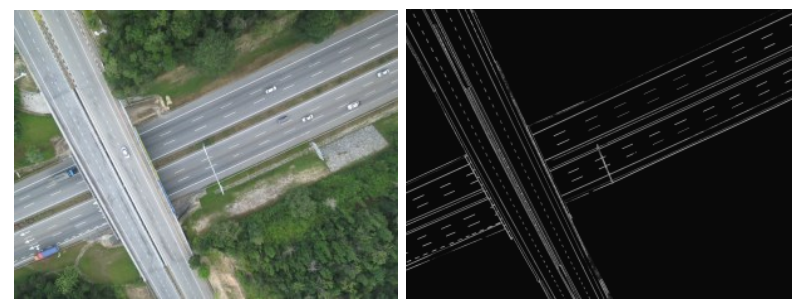

(c)

Fig. 7. Examples of UTeM images dataset with their corresponding ground truth images. (a) Main hall image, (b) mosque image and (c) highway image.

Table 1 displays the results in terms of values regarding the main hall image. In the context of $\mathrm{f}$ measure values, the Prewitt method provided a superior result in comparison to those attained by the Sobel, LoG and Canny methods. The average f-measure results acquired from all images in the UTeM UAVs dataset revealed that the performance of the Prewitt method surpasses those of the other methods. This is portrayed in Table 2.

As for the edge image, the results pointed to the fact that the amount of unnecessary edges generated is less in the Prewitt method than in the other methods. The performance of the Sobel method turned out second in this area, followed by the LoG method, while the Canny method was observed to generate the most unnecessary edges. These results are exhibited in Figure 5.

Table 1. Results derived from the main hall image

\begin{tabular}{|c|c|c|c|c|}
\hline & Sobel & Log & Canny & Prewitt \\
\hline Accuracy & 0.983708 & 0.952329 & 0.902309 & 0.983931 \\
\hline Precision & 0.110587 & 0.0376273 & 0.0238827 & 0.111776 \\
\hline Recall & 6.75975 & 5.74625 & 7.28475 & 6.7575 \\
\hline F-measure & 0.217614 & 0.074765 & 0.0476093 & 0.219921 \\
\hline
\end{tabular}

Table 2. Average results from the UTeM UAVs images dataset

\begin{tabular}{|c|c|c|c|c|}
\hline & Sobel & Log & Canny & Prewitt \\
\hline Accuracy & 0.986759 & 0.954141 & 0.912615 & 0.986947 \\
\hline Precision & 0.256592 & 0.110509 & 0.086257 & 0.257869 \\
\hline Recall & 18.06275 & 15.95981 & 21.54747 & 17.99098 \\
\hline F-measure & 0.505928 & 0.219475 & 0.171797 & 0.508382 \\
\hline
\end{tabular}




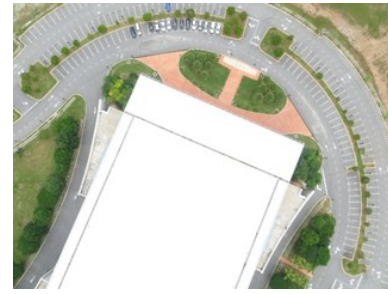

(a)

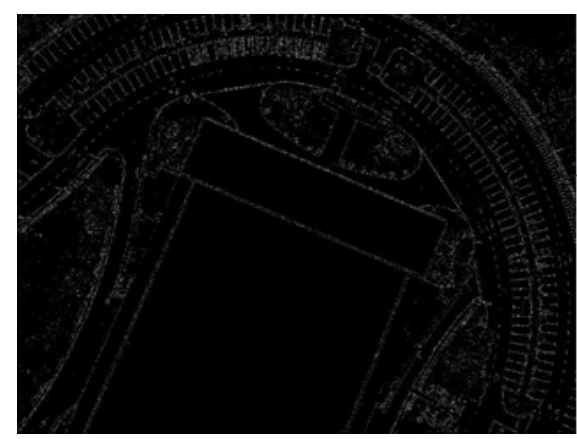

(c)

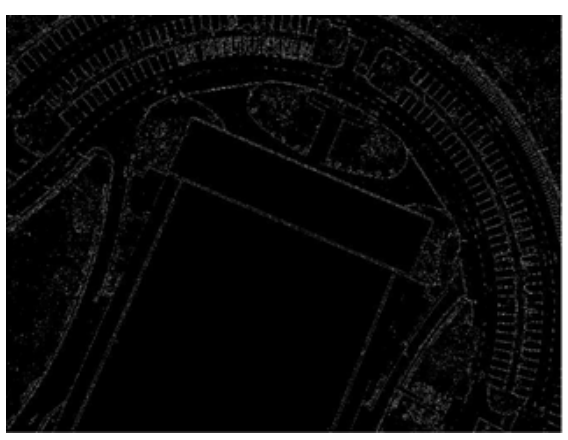

(d)

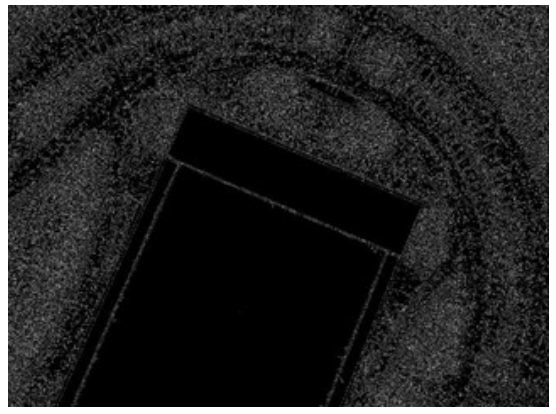

(e)

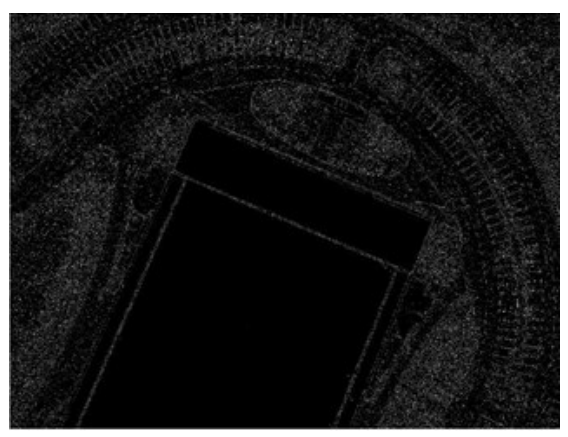

(f)

Fig. 5. The acquired image results. (a) Original main hall image, (b) ground truth image, (c) image acquired from Sobel method, (d) image acquired from Prewitt method, (e) image acquired from Canny method and (f) image acquired from LoG method.

\section{Conclusions}

The results of the experiment have shown that the Prewitt methods outperform other methods in detecting edges for UTeM UAVs image dataset. In fact, Prewitt and Sobel methods have produced approximately the same accuracy. Both of these methods are generally good at detecting edges and their orientations. Canny method gave the most unwanted edges compared to other methods. This is because one of the drawbacks of Canny method, it is sensitive towards weak edges. The UTeM UAVs image dataset is open for comparison on any other techniques and can be used by any researchers. It is hope that the UTeM UAVs image dataset can serve as a reference benchmark in edge detection area and thus will lead to a valid objective evaluation in edge detection. This can eventually support the progression of research work in edge detection for future ahead.

The deepest gratitude and thanks to Universiti Teknikal Malaysia Melaka (UTeM) in supporting this research.

\section{References}

1. S. E. Umbaugh, Digital image processing and analysis: human and computer vision applications with CVIPtools, Second Edi. CRC Press, 2010.

2. L. Mulrane, E. Rexhepaj, S. Penney, J. J. Callanan, and W. M. Gallagher, "Automated image analysis in histopathology: a valuable tool in medical diagnostics," Expert Rev. Mol. Diagn., vol. 8, no. 6, pp. 707-725, Nov. 2008.

3. H. Zhou, H. Kong, L. Wei, D. Creighton, and S. Nahavandi, "On Detecting Road Regions in a Single UAV Image," IEEE Trans. Intell. Transp. Syst., vol. 18, no. 7, pp. 1713-1722, 2016.

4. M. A. M. Abdullah, S. S. Dlay, W. L. Woo, and J. A. Chambers, "Robust Iris Segmentation Method Based on a New Active Contour Force With a Noncircular Normalization," IEEE Trans. Syst. Man, Cybern. Syst., pp. 1-14, 2016.

5. [5] P. Smith, D. B. Reid, C. Environment, L. Palo, P. Alto, and P. L. Smith, "Otsu1975," vol. 20, no. 1, pp. 62-66, 1979.

6. A. Lucieer, D. Turner, D. H. King, and S. A. Robinson, "Using an Unmanned Aerial Vehicle (UAV) to capture micro-topography of Antarctic moss beds," Int. J. Appl. Earth Obs. Geoinf., vol. 27, pp. 53-62, Apr. 2014. 
7. T. Y. Lim and M. M. Ratnam, "Edge detection and measurement of nose radii of cutting tool inserts from scanned 2-D images," Opt. Lasers Eng., vol. 50, no. 11, pp. 1628-1642, Nov. 2012.

8. J. Huang, X. You, Y. Y. Tang, L. Du, and Y. Yuan, "A novel iris segmentation using radialsuppression edge detection," Signal Processing, vol. 89, no. 12, pp. 2630-2643, 2009.

9. S. a. Coleman, B. W. Scotney, and S. Suganthan, "Multi-scale edge detection on range and intensity images," Pattern Recognit., vol. 44, no. 4, pp. 821-838, Apr. 2011.

10. W. H. W. He and K. Y. K. Yuan, An improved Canny edge detector and its realization on FPGA. Ieee, 2008, pp. 6561-6564.

11. S. B. Kutty, S. Saaidin, P. N. A. Megat Yunus, and S. Abu Hassan, "Evaluation of canny and sobel operator for logo edge detection," ISTMET 2014 - 1st Int. Symp. Technol. Manag. Emerg. Technol. Proc., vol. 2, no. Istmet, pp. 153-156, 2014.

12. Z. Othman, A. Abdullah, and A. S. Prabuwono, "A statistical Approach of Multiple Resolution Levels for Canny Edge Detection," in Intelligent Systems Design and Applications (ISDA), 2012, 2012, pp. 837-841.
13. Z. Othman and A. Abdullah, "An Adaptive Threshold Based On Multiple Resolution Levels for Canny Edge Detection," in IRICT 2017: Recent Trends in Information and Communication Technology, 2017, pp. 316323.

14. W. Gao, L. Yang, X. Zhang, and H. Liu, "An improved Sobel edge detection," Proc. - 2010 3rd IEEE Int. Conf. Comput. Sci. Inf. Technol. ICCSIT 2010, vol. 5, pp. 67-71, 2010.

15. J. Canny, "A computational approach to edge detection.," IEEE Trans. Pattern Anal. Mach. Intell., vol. 8, no. 6, pp. 679-98, Jun. 1986.

16. B. Wang and S. Fan, "An improved CANNY edge detection algorithm Bing," 2009 Second Int. Work. Comput. Sci. Eng., 2009.

17. G. Jie and L. Ning, "An Improved Adaptive Threshold Canny Edge Detection Algorithm," 2012 Int. Conf. Comput. Sci. Electron. Eng., pp. 164-168, Mar. 2012.

18. Nasruddin Abu Sari, Asmala Ahmad, MY Abu Sari, S Sahib, AW Rasib (2015) Development Of Rapid Low-Cost LARS Platform For Oil Palm Plantation. Jurnal Teknologi, 77 (20), 99 105 (Scopus). EISSN 2180-3722. 